\title{
FATOU THEOREMS ON DOMAINS IN $\mathbf{C}^{n}$
}

\author{
STEVEN G. KRANTZ
}

If $D \subseteq \mathbf{C}$ is the unit disc and $0<p<\infty$ then define $H^{p}(D)$ to be those $f$ holomorphic on $D$ such that

$$
\|f\|_{H^{p}} \equiv \sup _{0<r<1} \int_{0}^{2 \pi}\left|f\left(r e^{i \theta}\right)\right|^{p} d \theta^{1 / p}<\infty .
$$

The space $H^{\infty}(D)$ consists of the bounded holomorphic functions equipped with the supremum norm. The classical Fatou theorem asserts that if $f \in$ $H^{p}(D)$ then for a.e. (with respect to linear measure in $\left.\partial D\right) e^{i \theta} \in \partial D$ it holds that

$$
\lim _{r \rightarrow 1^{-}} f\left(r e^{i \theta}\right) \equiv f^{*}\left(e^{i \theta}\right)
$$

exists. For $p \geq 1$, the function $f$ can be recovered from $f^{*}$ by means of the Cauchy or Poisson integral formulas. See [JG or SK].

It is an important and useful fact that this radial approach to $e^{i \theta} \in \partial D$ may be replaced by a more general type of approach: if $\alpha>1$ and $P \in \partial D$ we define the Stolz region

$$
\Gamma_{\alpha}(P)=\{z \in D:|z-P|<\alpha \cdot(1-|z|)\} .
$$

Then, for any $0<p \leq \infty, f \in H^{p}(D)$, and $\alpha>1$ we have

$$
\lim _{\Gamma_{\alpha}(P) \ni z \rightarrow P} f(z)=f^{*}(P)
$$

for a.e. $P \in \partial D$. It is known [IP] that this nontangential method of approach is best possible.

If $\Omega \subseteq \mathbf{C}^{n}$ is a smoothly bounded domain then there is a similar theory of $H^{p}$ spaces (also classical). (Let $\delta_{\Omega}(z) \equiv \operatorname{dist}(z, \partial \Omega)$ ). In this theory one replaces

(i) the circles $\left\{r e^{i \theta}: 0 \leq \theta<2 \pi\right\}$ by $\partial \Omega_{\varepsilon} \equiv\left\{z \in \Omega\right.$ : $\left.\delta_{\Omega}(z)=\varepsilon\right\}, \varepsilon$ small;

(ii) linear measure by $(2 n-1)$-dimensional area measure;

(iii) Stolz regions $\Gamma_{\alpha}$ by cones in space of fixed aperture.

It is a remarkable discovery of Korányi (for the ball and for certain symmetric domains [AK1, AK2]) and of Stein (for smoothly bounded domains [ES1]) that in $\mathbf{C}^{n}, n>1$, the conical approach regions are not optimal for studying the boundary behavior of $H^{p}$ functions. Indeed, they may be replaced by admissible approach regions which are conical in "complex normal directions" and parabolic in "complex tangential directions" (see [ES1, SK]).

Received by the editors June 30, 1986.

1980 Mathematics Subject Classification. Primary 32A35; Secondary 42B30.

Work supported in part by a grant from the National Science Foundation. I am grateful to the Institute for Advanced Study for its hospitality during a portion of this work. 
An even more recent development is that, for many domains $\Omega$ in $\mathbf{C}^{n}$, even the admissible approach regions are not optimal. The correct shape for the approach regions at a point $P \in \partial \Omega$ seems to be connected to the Levi geometry of $\partial \Omega$ at $P$. The paper $[\mathbf{C K}]$ contains Lindelöf theorems (pointwise results) which suggest what the optimal approach regions ought to be, while [NSW] performs the much more difficult task of explicitly calculating the correct approach regions for a.e. results on the (very special) domains of finite type in $\mathbf{C}^{2}$.

We announce here a rather general differential geometric method for studying the matters discussed above. This method gives a canonical (and, in all calculable instances, optimal) method to assign approach regions to the boundary points of a smooth $\Omega \subseteq \mathbf{C}^{n}$, thus at least partially answering a question posed by Stein in [ES2].

For specifics, let $\Omega \subseteq \mathbf{C}^{n}$ be a smoothly bounded domain. Let $\rho$ be a metric on $\Omega$ (Riemannian, Finsler, or otherwise) and let $d V$ be an associated volume (there is considerable freedom in the choice of $d V$ ). We make two hypotheses about the compatibility of $\rho$ and $d V$ with the complex structure on $\Omega$ :

(a) There is a $C>0$ such that if $B(P, r) \subseteq \Omega$ is a metric ball and $f \in H^{p}(\Omega)$ then

$$
|f(P)|^{p} \leq \frac{C}{V(B(P, r))} \int_{B(P, r)}|f(\zeta)|^{p} d V(\varsigma) .
$$

(b) If $z, w \in \Omega$ are inside a tubular neighborhood of $\partial \Omega$ and have the same Euclidean normal projection to $\partial \Omega\left(\pi_{\Omega} z=\pi_{\Omega} w\right)$ and if $\alpha>0$ and $\operatorname{dis}_{\Omega}(z, \Omega) \geq \operatorname{dis}_{\Omega}(w, \Omega)$ (this is Euclidean distance) then

$$
\pi_{\Omega}(B(z, \alpha)) \supseteq \pi_{\Omega}(B(w, \alpha)) .
$$

We discuss the plausibility of these hypotheses at the end of this announcement.

The thrust of our metric point of view is to define our approach regions, boundary measures, and balls in the boundary in terms of $\rho$ and $d V$. If $P \in \partial \Omega$, let $\nu_{P}$ be the unit outward normal to $\partial \Omega$ at $P$. Let $\alpha>0$ be fixed once and for all and define the corresponding approach region at $P$ to be

$$
\mathfrak{M}_{\alpha}(P)=\left\{z \in \Omega: \rho\left(z,-\nu_{P}\right)<\alpha\right\} .
$$

Here, in the expression $\rho\left(z,-\nu_{P}\right)$, the symbol $-\nu_{P}$ is interpreted to denote a set of points. Now we use the Carathéodory measure construction (see $[\mathbf{H F}]$ ) to define a new measure on $\partial \Omega$. For each $P \in \partial \Omega, r>0$ small, we define

$$
\beta_{\alpha}(P, r) \equiv \pi\left(B\left(P-r \nu_{P}, \alpha\right)\right)
$$

where, as usual, $B(\cdot, \cdot)$ denotes a $\rho$-metric ball in $\Omega$ and $\nu_{P}$ is once again a vector. Let $\omega(r), 0 \leq r<\infty$, be an increasing function with $\omega(0)=0$. If $\delta>0$ and $E \subseteq \partial \Omega$ define

$$
\mu_{\delta}(E) \equiv \inf _{C_{\delta}(E)} \sum_{i} \omega\left(r_{i}\right) V\left(B\left(P_{i}-r_{i} \nu_{P_{i}}, \alpha\right)\right)
$$

Here $C_{\delta}(E)$ is the collection of countable coverings $\left\{\beta\left(P_{i}, r_{i}\right)\right\}$ of $E$ by balls in $\partial \Omega$ with all $r_{i} \leq \delta$. Now $\mu_{\delta}(E)$ increases as $\delta \rightarrow 0$, so we may define

$$
\mu(E) \equiv \lim _{\delta \rightarrow 0^{+}} \mu_{\delta}(E)
$$


As is standard in the theory of spaces of homogeneous type, we must hypothesize that $0<\mu(U)<\infty$ for any open $U \subseteq \partial \Omega$.

THEOREM. Let $f \in H^{p}(\Omega), 0<p \leq \infty$. Then for $\mu$-almost every $P \in \partial \Omega$ it holds that

$$
\lim _{\mathfrak{M}_{\alpha}(P) \ni z \rightarrow P} f(z)=f^{*}(P) .
$$

One natural choice of $\rho, V, \omega$ is to let $\rho$ be one of the Bergman, Carathéodory, or Kobayashi metrics (see [SK]), to let $d V$ be a suitable associated volume, and to let $\omega(r)=r^{k}$, where $k$ is a suitably chosen exponent. Then the following assertions hold:

(1) The constructions of $\mathfrak{M}_{\alpha}, d \mu$ are invariant under biholomorphic maps which extend $C^{1}$ to the boundary.

(2) Hypotheses (a) and (b) hold on the disc (with $k=1$ ), the ball (with $k=n$ ), strongly pseudoconvex domains (with $k=n$ ), domains of finite type in $\mathbf{C}^{2}$ (with $k=2$ ), convex domains (with $k=n$ ), and near strongly psuedoconcave points (with $k=2 n-1$ ).

(3) Near strongly pseudoconvex points (which are generic), $d \mu$ is mutually absolutely continuous with respect to the usual boundary area measure.

It is known [GA] that on the disc, the ball, strongly pseudoconvex domains, and domains of finite type in $\mathbf{C}^{2}$, the approach regions $\mathfrak{M}_{\alpha}$ constructed with the Kobayashi or Bergman metrics are comparable to those defined in [SK, AK1, ES1, and NSW] respectively. At a strongly pseudoconcave point, $\mathfrak{M}_{\alpha}(P)$ contains a set of the form $\left\{z \in \Omega:|z-P|<s_{0}\right\}, s_{0}$ small. Thus approach at such a boundary point is unrestricted (as it should be, by the Hartogs extension phenomenon or Kugelsatz).

Other choices of $\omega$ can be used to pick out special curves in $\partial \Omega$. For instance, the choice $\omega(r)=r$ yields, near strongly pseudoconvex points and points of finite type in $\mathbf{C}^{2}$, a measure which restricts to linear measure on complex normal curves and to zero measure on other curves. Our theory extends, by means of this measure, to recover a version of the Nagel-Rudin theorem [NR] on boundary behavior of $H^{\infty}$ functions along complex normal curves.

We believe that hypotheses (a) and (b), or suitable variants, are true essentially all the time. Using $L^{2}$ estimates for the $\bar{\partial}$ problem, one can verify (a variant of) (b) for any bounded, smooth pseudoconvex domain. A slightly weakened (but sufficient for our purposes) version of (b) is easily checked for the Kobayashi metric on any smoothly bounded domain. Hypothesis (a) is more difficult: it holds (for any of the standard invariant metrics $\rho$ and for suitable $d V$ ) on the disc, ball, polydisc, strongly pseudoconvex domains, on convex domains, near strongly pseudoconcave points, and near points of finite type in $\mathbf{C}^{2}$. However the calculations in the latter instance are quite difficult and require the ideas of [NSW]. The crux of our approach is not to eliminate calculation (indeed this seems impossible) but instead to unify known results and to relegate the calculations to the arena of calculating a metric.

It should be stressed that we do not know how to derive our results by using the methods of, or even the language of, spaces of homogeneous type. 
The covering lemma which we ultimately prove is inspired by those in $[\mathbf{H F}$, KP, EGKP], relies on dimension and on the metric, and is independent of any measure. It hinges on the following fact: There is a constant $N>0$ such that if $B_{i} \equiv B\left(z_{i}, \alpha\right)$ is a collection of metric balls in $\Omega$ such that $\left\{\pi\left(B_{i}\right)\right\}$ covers a compact set $E \subseteq \partial \Omega$, then there is a subcollection $\mathfrak{B}=\left\{B_{i_{j}}\right\}$ whose projections still cover $E$ such that $\mathfrak{B}$ partitions into $N$ families $\left\{\mathfrak{B}_{l}\right\}_{l=1}^{N}$ of balls and the projections to $\partial \Omega$ of the elements of $\mathfrak{B}_{l}$, each $l$, are pairwise disjoint. This easily gives a Besicovitch-type covering lemma for the balls $\beta(\cdot, \cdot)$ in the boundary.

Details of the proof of the theorem will appear elsewhere. We also have obtained results about the Nevanlinna class (using ideas in $[\mathbf{R B}]$ ), atomic theory (using ideas in [MW]), and the equivalence of $\mathfrak{M}_{\alpha}$-boundedness and the existence of $\mathfrak{M}_{\alpha}$ limits. We intend to develop the theory of the Lusin area integral in the metric framework as well.

\section{REFERENCES}

[GA] G. Aladro, thesis, Pennsylvania State University, 1985.

[RB] S. R. Barker, Two theorems on boundary values of analytic functions, Proc. Amer. Math. Soc. 68 (1978), 48-54.

[CK] J. A. Cima and S. G. Krantz, The Lindelöf principle and holomorphic functions of several complex variables, Duke Math. J. 50 (1983), 303-326.

[EGKP] P. Erdős, C. Godsil, S. G. Krantz, and T. D. Parsons, Intersection graphs for families of balls, preprint.

[HF] H. Federer, Geometric measure theory, Springer-Verlag, Berlin and New York, 1969.

[JG] J. B. Garnett, Bounded analytic functions, Academic Press, New York, 1981.

[PK] P. Koosis, Sommabilité de la fonction maximale et appartenance a $H_{1}$. Cas de plusiers variables, C. R. Acad. Sci. Paris 288 (1979), 489-492.

[AK1] A. Korányi, Harmonic functions on Hermitian hyperbolic space, Trans. Amer. Math. Soc. 135 (1969), 507-516.

[AK2] _ Poisson integrals and boundary components of symmetric spaces, Invent. Math. 34 (1976), 19-35.

[SK] S. G. Krantz, Function theory of several complex variables, John Wiley and Sons, New York, 1982.

[KP] S. G. Krantz and T. D. Parsons, Antisocial subcoverings of self-centered covers, Amer. Math. Monthly 93 (1986), 45-48.

[NR] A. Nagel and W. Rudin, Local boundary behavior of bounded holomorphic functions, Canad. J. Math. 30 (1978), 583-592.

[NSW] A. Nagel, E. M. Stein, and S. Wainger, Boundary behavior of functions holomorphic in domains of finite type, Proc. Nat. Acad. Sci. U.S.A. 78 (1981), 6596-6599.

[IP] I. Privalov, Randeigenschaften Analytischer Funktionen, Deutsch Verlag der Wissenschaften, Berlin, 1956.

[ES1] E. M. Stein, Boundary behavior of holomorphic functions of several complex variables, Princeton Univ. Press, Princeton, N.J., 1972.

[ES2] _ Some problems in harmonic analysis, Proc. Sympos. Pure Math., vol. 35, Amer. Math. Soc., Providence, R.I., pp. 3-20.

[MW] J. Michael Wilson, A simple proof of the atomic decomposition for $H^{p}\left(\mathbf{R}^{n}\right)$, $0<p \leq 1$, Studia Math. 74 (1982), 25-33.

Department of Mathematics, Pennsylvania State University, UniverSITY PARK, PENNSYLVANIA 16802

Current address: Washington University, St. Louis, Missouri 63130 